\title{
Indicações para ceratoplastia penetrante no Hospital das Clínicas-UNICAMP
}

\author{
Penetrating keratoplasty indications in "Hospitaldas Clínicas-UNICAMP"
}

\author{
Vanessa Gonçalves Crespi Flores ${ }^{1}$ \\ Helena Luísa Reimer Dias ${ }^{2}$ \\ Rosane Silvestre de Castro ${ }^{3}$
}

Trabalho realizado no Setor de Córnea do Departamento de Oftalmologia da Universidade Estadual de Campinas - UNICAMP - Campinas (SP) - Brasil.

Médica Oftalmologista da Universidade Estadual de Campinas - UNICAMP - Campinas (SP) - Brasil

${ }^{2}$ Médica Oftalmologista da UNICAMP - Campinas (SP) - Brasil.

${ }^{3}$ Doutora em Oftalmologia pela Faculdade de Ciências Médicas da UNICAMP - Campinas (SP) - Brasil.

Endereço para correspondência: Vanessa Gonçalves Crespi Flores. Rua Alberto Macchi, 40 - Apto. 101D Campinas (SP) CEP 13094-525

E-mail:vanweb@uol.com.br

Recebido para publicação em 25.07.2005

Última versão recebida em 30.11.2006

Aprovação em 13.12.2006

Nota Editorial: Depois de concluída a análise do artigo sob sigilo editorial e com a anuência da $\mathrm{Dr}^{\mathrm{a}}$. Adriana dos Santos Forseto e do Dr. José Beniz Neto sobre a divulgação de seus nomes como revisores, agradecemos suas participações neste processo.

\begin{tabular}{|c|}
\hline RESUMO \\
\hline $\begin{array}{l}\text { Objetivo: Determinar as principais causas de indicação de transplante } \\
\text { penetrante no Hospital das Clínicas-UNICAMP no período de janeiro de } \\
1999 \text { a dezembro de } 2003 \text {. Métodos: Estudo de série de casos, retrospectivo, } \\
\text { não comparativo. Os autores revisaram os prontuários de } 857 \text { pacientes } \\
\text { submetidos à ceratoplastia penetrante no Hospital das Clínicas-UNICAMP } \\
\text { entre } 1999-2003 \text { e os classificaram em categorias diagnósticas de indicação } \\
\text { para cirurgia. Resultados: Dentre os } 857 \text { prontuários revisados a idade } \\
\text { variou de } 0-88 \text { anos (média } 44 \text { anos } 11,2) \text {. Dentre as principais causas de } \\
\text { indicação de transplante de córnea encontramos: ceratocone em } 427 \text { casos } \\
\text { (49,82\%); úlcera de córnea infecciosa perfurada ou não, } 152 \text { casos }(17,74 \%) \text {; } \\
\text { falência de transplante prévio, } 87 \text { casos ( } 10,15 \%) \text {; ceratopatia bolhosa, } 72 \\
\text { casos ( } 8,40 \%) \text { distrofia de Fuchs, } 59 \text { casos }(6,88 \%) \text {; seqüela de tracoma, } \\
28 \text { casos (3,27\%); outras causas, } 32 \text { casos }(3,74 \%) \text { Entre as crianças até } \\
10 \text { anos a principal causa de indicação de transplante foram as úlceras } \\
\text { infecciosas (77,78\%) e entre } 11-50 \text { anos o ceratocone foi a principal causa } \\
(71,65 \%) \text {. Conclusões: Este estudo foi composto por uma população } \\
\text { jovem eas principais causas de indicação de transplante foram oceratocone } \\
\text { e os transplantes tectônicos. }\end{array}$ \\
\hline
\end{tabular}

Descritores: Doenças da córnea/cirurgia; Transplante de córnea; Ceratoplastia penetrante; Ceratite

\section{INTRODUÇÃO}

A cirurgia de transplante de órgãos vem se difundindo nas últimas décadas e firmando-se como um dos grandes avanços da medicina. Dentre os transplantes, o de córnea é o mais freqüente devido às facilidades técnicas e ao número de órgãos doados ${ }^{(1)}$. As indicações do transplante de córnea podem ser: óptica ou funcional, tectônica ou reconstrutiva, terapêutica e cosmética ${ }^{(2)}$.

Thomas (1955) atribuiu o primeiro transplante de córnea a Pellier de Quengsy (1789), que substituiu a córnea por uma prótese de vidro, e a Zirm (1906) o primeiro transplante com sucesso após oito meses. Na década de 30, Filatov descreveu os primeiros transplantes de córnea utilizando olhos de cadáver como doadores ${ }^{(3)}$.

Segundo dados da OMS (2001) ${ }^{(4)}$, utilizando como definição de cegueira visão $\leq 3 / 60$, é estimado que existam no mundo cerca de 45 milhões de indivíduos cegos bilaterais e outros 135 milhões que têm diminuição grave da visão. As doenças corneanas ficam em segundo lugar como causa de cegueira, atrás apenas da catarata, mas sua epidemiologia é mais complicada, pois sua etiologia tem uma série de variações como infecções e doenças inflamatórias. 
Por outro lado, a prevalência de doenças corneanas varia de país para país, e de população para população, dependendo de muitos fatores, entre eles as condições de saúde pública a que a população está sujeita ${ }^{(5)}$.

Em países em desenvolvimento os leucomas correspondem a $40 \%$ dos casos de cegueira unilateral em crianças $^{(6)}$.

Com o aumento do número de cirurgias de catarata realizadas com implante intra-ocular nos últimos anos, aumentou muito o número de casos de descompensação corneana e a necessidade de realização de transplante de córnea nesses $\operatorname{casos}^{(7-8)}$.

Este trabalho tem por objetivo identificar as principais causas de indicação de transplante de córnea no Hospital das Clínicas-UNICAMP no período de 1999 a 2003.

\section{MÉTODOS}

Foi realizado estudo de série de casos não comparativo, retrospectivo, dos prontuários de 857 pacientes submetidos a transplante penetrante de córnea no Hospital das ClínicasUNICAMP no período de 1999 a 2003, sendo avaliados o diagnóstico para indicação da cirurgia e características pessoais dos pacientes transplantados (idade e sexo).

Foram realizados 857 transplantes por médicos residentes do $3^{\circ}$ ano de residência médica em oftalmologia, supervisionados por médico assistente, utilizando a mesma técnica cirúrgica.

Em todos os casos as cirurgias foram realizadas sob anestesia geral. Os trépanos utilizados variaram entre 7,25 e 8,50 mm, com diferença de 0,25 - 0,50 mm entre doador e receptor. Em todos os olhos foi realizado o procedimento cirúrgico de ceratoplastia penetrante, sendo a sutura do botão realizada com 16 pontos interrompidos de fio nylon monofilamentar 10-0. Ao final das cirurgias foi realizada injeção subconjuntival de dexametasona a $4 \mathrm{mg} / \mathrm{ml}$ e gentamicina $20 \mathrm{mg} / \mathrm{ml}$. Nas primeiras 24 horas os pacientes permaneceram internados, em repouso relativo, com curativo oclusivo. Os pacientes tiveram alta hospitalar após avaliação oftalmológica na qual apresentaram: Seidel negativo, pontos íntegros, câmara anterior formada e ausência de sinais infecciosos.

Os diagnósticos para indicação das cirurgias foram classificados em ordem decrescente de incidência, e foram relacionadas as incidências de cada diagnóstico por faixa etária.

Os resultados foram descritos em tabelas de freqüência.

\section{RESULTADOS}

Dos 857 transplantes realizados nesse período, 417 pacientes eram do sexo feminino $(48,72 \%)$ e 439 do sexo masculino $(51,28 \%)$. A idade dos pacientes variou de $0-88$ anos, sendo a média de 44 anos $( \pm 1,2)$.

Com relação às indicações dos transplantes: ceratocone foi responsável por 427 casos (49,82\%); úlcera infecciosa com perfuração ou não, 152 casos $(17,74 \%)$; falência de transplante prévio, 87 casos $(10,15 \%)$; ceratopatia bolhosa, 72 casos $(8,40 \%)$; distrofia de Fuchs, 59 casos $(6,88 \%)$; seqüela de tracoma, 28 casos (3,27\%); anomalias congênitas, 5 casos (0,58\%); leucoma pós-herpes e pós-úlcera, 4 casos cada $(0,47 \%)$; e outras indicações, 19 casos $(2,22 \%)$ (Tabela 1$)$.

Segundo a faixa etária as indicações mais frequientes foram: de 0-10 anos, úlcera corneana (7 casos - 77,78\%); de 11-50 anos, ceratocone (417 casos - 71,65\%); de 51-60 anos úlcera corneana, (19 casos - 29,23\%); de 61-70 anos, distrofia de Fuchs (30 casos $27,03 \%$ ); de 71-80 anos, ceratopatia bolhosa (26 casos - 36,62\%); acima de 80 anos, úlcera corneana (9 casos - 47,37\%) (Tabela 2).

O ceratocone foi a principal causa de indicação de transplante corneano nas idades de 11 a 50 anos, e na faixa etária de 21-30 anos correspondeu a 81,34\%.

\section{DISCUSS ÃO}

Em nossa pesquisa, foi feito um estudo de série de casos não comparativo, retrospectivo e descritivo. Desta forma, a relação entre as causas e os resultados é limitada.

A principal causa de indicação de ceratoplastia penetrante neste estudo foi o ceratocone com aproximadamente $50 \%$ das indicações. Em trabalhos com média de idade maior que 50 anos as indicações para ceratocone foram ao redor de $10-15 \%{ }^{(8-9)}$. Tal diferença pode ser atribuída ao fato de o ceratocone ser mais freqüente na população mais jovem.

$\mathrm{Na}$ literatura vários autores referem à ceratopatia bolhosa após cirurgia de catarata como a principal causa de indicação de transplante de córnea, com dados que variam de 20-30\%, tendo aumentado em cinco vezes na década de $80 \mathrm{com}$ a popularização da facoemulsificação e o aumento dos implantes intra-oculares ${ }^{(7-8,10)}$.

Nesse estudo a ceratopatia bolhosa pós-cirurgia de catarata apareceu em $4^{\circ}$ lugar com aproximadamente $8 \%$ das indicações. O que se deve levar em consideração é que a população estudada teve média de idade de 44 anos e a indicação de cirurgia de catarata nesta faixa etária é menor.

Em crianças, a principal causa de cegueira monocular está associada a doenças corneanas, principalmente relacionadas a doenças endêmicas como tracoma, sendo relatado incidência 20 vezes maior em países da África e Ásia do que em países

\begin{tabular}{|lcc|}
\hline \multicolumn{3}{|c|}{$\begin{array}{c}\text { Tabela 1. Causas de indicação de transplante de córnea no Hospital } \\
\text { das Clínicas-UNICAMP }\end{array}$} \\
$\begin{array}{l}\text { (1999-2003) } \\
\text { Indicação do transplante }\end{array}$ & $\mathbf{n}$ & $\mathbf{f ( \% )}$ \\
\hline 1. Ceratocone & 427 & 49,82 \\
2. Úlcera perfurada ou não & 152 & 17,74 \\
3. Falência de transplante prévio & 87 & 10,15 \\
4. Ceratopatia bolhosa & 72 & 8,40 \\
5. Distrofia de Fuchs & 59 & 6,88 \\
6. Seqüela de tracoma & 28 & 3,27 \\
7. Anomalias congênitas & 5 & 0,58 \\
8. Leucoma pós-herpes & 4 & 0,47 \\
9. Leucoma pós-úlcera & 4 & 0,47 \\
10. Outras & 19 & 2,22 \\
Total & 857 & 100,00 \\
\hline
\end{tabular}




\begin{tabular}{|c|c|c|c|c|c|c|c|c|c|c|}
\hline \multirow[t]{2}{*}{ Causa* } & \multicolumn{10}{|c|}{ Idade (anos) } \\
\hline & & $\leq 10$ & $11-20$ & $21-30$ & $31-40$ & $41-50$ & $51-60$ & $61-70$ & $71-80$ & $>80$ \\
\hline \multirow[t]{2}{*}{ 1. Ceratocone } & $\mathrm{n}$ & 0 & 154 & 170 & 57 & 36 & 7 & 1 & 2 & 0 \\
\hline & $f(\%)$ & 0 & 86,52 & 81,34 & 61,29 & 35,29 & 10,77 & 0,90 & 2,82 & 0 \\
\hline \multirow[t]{2}{*}{ 2. Úlcera perfurada ou não } & $\mathrm{n}$ & 7 & 16 & 16 & 16 & 32 & 19 & 29 & 8 & 9 \\
\hline & $f(\%)$ & 77,78 & 8,99 & 7,66 & 17,20 & 31,37 & 29,23 & 26,13 & 11,27 & 47,37 \\
\hline \multirow{2}{*}{$\begin{array}{l}\text { 3. Falência de transplante } \\
\text { prévio }\end{array}$} & $\mathrm{n}$ & 1 & 7 & 15 & 9 & 20 & 14 & 14 & 6 & 1 \\
\hline & $f(\%)$ & 11,11 & 3,93 & 7,18 & 9,68 & 19,61 & 21,54 & 12,61 & 8,45 & 5,26 \\
\hline \multirow[t]{2}{*}{ 4. Ceratoplastia bolhosa } & $\mathrm{n}$ & 0 & 0 & 0 & 2 & 3 & 9 & 27 & 26 & 5 \\
\hline & $f(\%)$ & 0 & 0 & 0 & 2,15 & 2,94 & 13,85 & 24,32 & 36,62 & 26,32 \\
\hline \multirow{2}{*}{ 5. Distrofia de Fuchs } & $\mathrm{n}$ & 0 & 0 & 0 & 3 & 2 & 7 & 30 & 14 & 3 \\
\hline & $f(\%)$ & 0 & 0 & 0 & 3,23 & 1,96 & 10,77 & 27,03 & 19,72 & 15,79 \\
\hline \multirow[t]{2}{*}{ 6. Seqüela de tracoma } & $\mathrm{n}$ & 0 & 0 & 0 & 0 & 0 & 6 & 8 & 14 & 0 \\
\hline & $f(\%)$ & 0 & 0 & 0 & 0 & 0 & 9,23 & 7,21 & 19,72 & 0 \\
\hline \multirow[t]{2}{*}{ 7. Anomalias congênitas } & $\mathrm{n}$ & 0 & 0 & 1 & 0 & 2 & 0 & 0 & 1 & 1 \\
\hline & $f(\%)$ & 0 & 0 & 0,48 & 0 & 1,96 & 0 & 0 & 1,41 & 5,26 \\
\hline \multirow[t]{2}{*}{ 8. Leucoma pós-herpes } & $\mathrm{n}$ & 0 & 0 & 1 & 1 & 1 & 0 & 1 & 0 & 0 \\
\hline & $f(\%)$ & 0 & 0 & 0,48 & 1,08 & 0,98 & 0 & 0,90 & 0 & 0 \\
\hline \multirow[t]{2}{*}{ 9. Leucoma pós-úlcera } & $\mathrm{n}$ & 0 & 0 & 0 & 1 & 2 & 1 & 0 & 0 & 0 \\
\hline & $f(\%)$ & 0 & 0 & 0 & 1,08 & 1,96 & 1,54 & 0 & 0 & 0 \\
\hline \multirow[t]{2}{*}{ 10. Outras } & $\mathrm{n}$ & 1 & 1 & 6 & 4 & 4 & 2 & 1 & 0 & 0 \\
\hline & $f(\%)$ & 11,11 & 0,56 & 2,87 & 4,30 & 3,92 & 3,08 & 0,90 & 0 & 0 \\
\hline \multirow[t]{2}{*}{ Total } & $\mathrm{n}$ & 9 & 178 & 209 & 93 & 102 & 65 & 111 & 71 & 19 \\
\hline & $f(\%)$ & 100,00 & 100,00 & 100,00 & 100,00 & 100,00 & 100,00 & 100,00 & 100,00 & 100,00 \\
\hline
\end{tabular}

industrializados ${ }^{(6)}$. Nossos resultados mostraram que os transplantes tectônicos em crianças abaixo de 10 anos corresponderam a aproximadamente $80 \%$ das indicações, estando associados principalmente a úlceras infecciosas.

Até pouco tempo trauma ocular e úlceras infecciosas não eram considerados fatores importantes como causa de cegueira na infância, já que ambos acometem principalmente um olho. Com o advento de campanhas em saúde pública que enfatizam a prevenção de cegueira em doenças endêmicas (como tracoma, oncocercoce e hipovitaminose) em países em desenvolvimento, as infecções corneanas e o trauma passaram a ter maior importância nessa faixa etária ${ }^{(4)}$.

Segundo Vanathi et al (2002) ${ }^{(11)}$ o transplante tectônico é um método efetivo para restaurar a integridade do olho em casos associados à infecção ou iminência de perfuração, mas o restabelecimento da visão não é tão efetivo, assim como a sobrevida do botão doador, pois são casos associados a muitas complicações pós-operatórias como sinéquias anteriores, glaucoma e recidiva da infecção.

Considerando que nesse estudo os transplantes tectônicos representaram cerca de $17 \%$, podemos inferir a magnitude do problema, pois a recuperação visual é difícil e esses pacientes muitas vezes chegam nessa situação por falta de informação ou acesso a tratamento adequado. Além disso, são pacientes que são submetidos à cirurgia em condições consideradas desfavoráveis. Os pacientes que necessitam ser submetidos a uma cirurgia de urgência, correm riscos cirúrgicos que poderiam ser evitados com tratamento precoce e adequado da infecção. Nosso serviço é centro de referência para tratamento de doenças corneanas, e por isso as indicações para transplantes tectônicos são mais freqüentes do que outros achados na literatura ${ }^{(11)}$.

A frequiência das indicações para transplante de córnea, entre as principais causas, tem mudado através das décadas e variam de país para país ${ }^{(12-13)}$; refletindo a mudança na incidência e tratamento das várias doenças corneanas assim como a incidência e efeitos dos procedimentos cirúrgicos oculares ${ }^{(14-15)}$. O tratamento precoce e adequado das infecções oculares pode reduzir o número de indicações de transplantes terapêuticos e com isso melhorar o prognóstico visual desses olhos.

Para melhor avaliar estas relações, em futuras pesquisas, estudos analíticos comparando causas, tratamentos e técnicas cirúrgicas utilizadas devem ser incentivados.

\section{CONCLUSÃO}

Neste estudo a média de idade foi de 44 anos e as principais causas de indicação de transplante foram o ceratocone e os transplantes tectônicos. Nos indivíduos menores que 10 anos foram as úlceras corneanas.

\section{ABSTRACT}

Purpose: To determine the main causes of penetrating keratoplasty indications at "Hospital das Clínicas-UNICAMP" (January, 1999 to December, 2003). Methods: A non-comparative, retrospective series of case studies. The authors reviewed the 
files of 857 patients who underwent penetrating keratoplasty at "Hospital das Clínicas-UNICAMP" between 1999-2003 and classified them into different categories according to diagnostic indication for surgery. Results: The age range was between 0 - 88 years (average 44 years \pm 1.2 ). The main causes of penetrating keratoplasty were: keratoconus in 427 cases $(49.82 \%) ; 152$ cases $(17.74 \%)$ of corneal ulceration (perforated or not); corneal graft failure in 87 cases $(10.15 \%)$; bullous keratopathy, 72 cases $(8.40 \%)$; Fuchs dystrophy in 59 cases $(6.88 \%)$; trachoma complications in 28 cases $(3.27 \%)$; other causes, 32 $(3.74 \%)$. In children under 10 years of age, the main cause of penetrating keratoplasty indications was infectious ulcer (77.78\%) and between 11-50 years of age, keratoconus was the main cause $(71.65 \%)$. Conclusion: This study was composed of a young population, and the main causes of penetrating keratoplasty were keratoconus and therapeutic keratoplasty.

Keywords: Corneal diseases/surgery; Corneal transplantation; Keratoplasty, penetrating; Keratitis

\section{REFERÊNCIAS}

1. Vail A, Gore SM, Bradley BA, Easty DL, Rogers CA, Armitage WJ. Influence of donor and histocompatibility factors on corneal graft outcome. Transplantation. 1994;58(11):1210-6.
2. Kanski JJ. Principles of keratoplasty In: Kanski JJ. Clinical Ophthalmology, a systematic approach. 3rd ed. London: Butterworth-Heinemann; 1994. p.140-2.

3. Filatov VP. Transplantation of the cornea. Arch Ophthalmol. 1935;13:321-47.

4. Whitcher JP, Srinivasan M, Upadhyay MP. Corneal blindness: a global perspective. Bull World Health Organ. 2001;79(3):214-21.

5. Smith GT, Taylor HR. Epidemiology of corneal blindness in developing countries. Refract Corneal Surg. 1991;7(6):436-9. Comment in: Refract Corneal Surg. 1991;7(6):417-8.

6. Jackson H, Foster A. Causes of blindness in northwest Cambodia. Ophthalmic Epidemiol. 1997;4(1):27-32. Comment in: Ophthalmic Epidemiol. 1997;4(1):1-2

7. Thompson RW Jr, Price MO, Bowers PJ, Price FW Jr. Long term graft survival after penetrating keratoplasty. Ophthalmology. 2003;110(7):1396-402.

8. Cosar CB, Sridhar MS, Cohen EJ, Held EL, Alvim PT, Rapuano CJ, et al. Indications for penetrating keratoplasty and associated procedures, 1996-2000. Cornea. 2002;21(2):148-51.

9. Dobbins KR, Price FW Jr, Whitson WE. Trends in the indications for penetrating keratoplasty in midwestern United States. Cornea. 2000;9(6):813-6.

10. Brady SE, Rapuano CJ, Arentsen JJ, Cohen EJ, Laibson PR. Clinical indications for and procedures associated with penetrating keratoplasty, 1983-1988. Am J Ophthalmol. 1989;108(2):118-22.

11. Vanathi M, Sharma N, Titiyal JS, Tandon R, Vajpayee RB. Tectonic grafts for corneal thinning and perforations. Cornea. 2002;21(8):792-7.

12. Mohamadi P, McDonnell JM, Irvine JA, McDonnell PJ, Rao N, Smith RE. Changing indications for penetrating keratoplasty, 1984-1988. Am J Ophthalmol. 1989;107(5):550-2.

13. Damji KF, Rootman J, White VA, Dubord PJ, Richards JS. Changing indications for penetrating keratoplasty in Vancouver, 1978-87. Can J Ophthalmol. 1990;25(5):243-8.

14. Arentsen JJ, Morgan B, Green WR. Changing indications for keratoplasty. Am J Ophthalmol. 1976;81(3):313-8.

15. Haamann $\mathrm{P}$, Jensen $\mathrm{OM}$, Schmidt $\mathrm{P}$. Changing indications for penetrating keratoplasty. Acta Ophthalmol (Copenh). 1994;72(4):443-6.

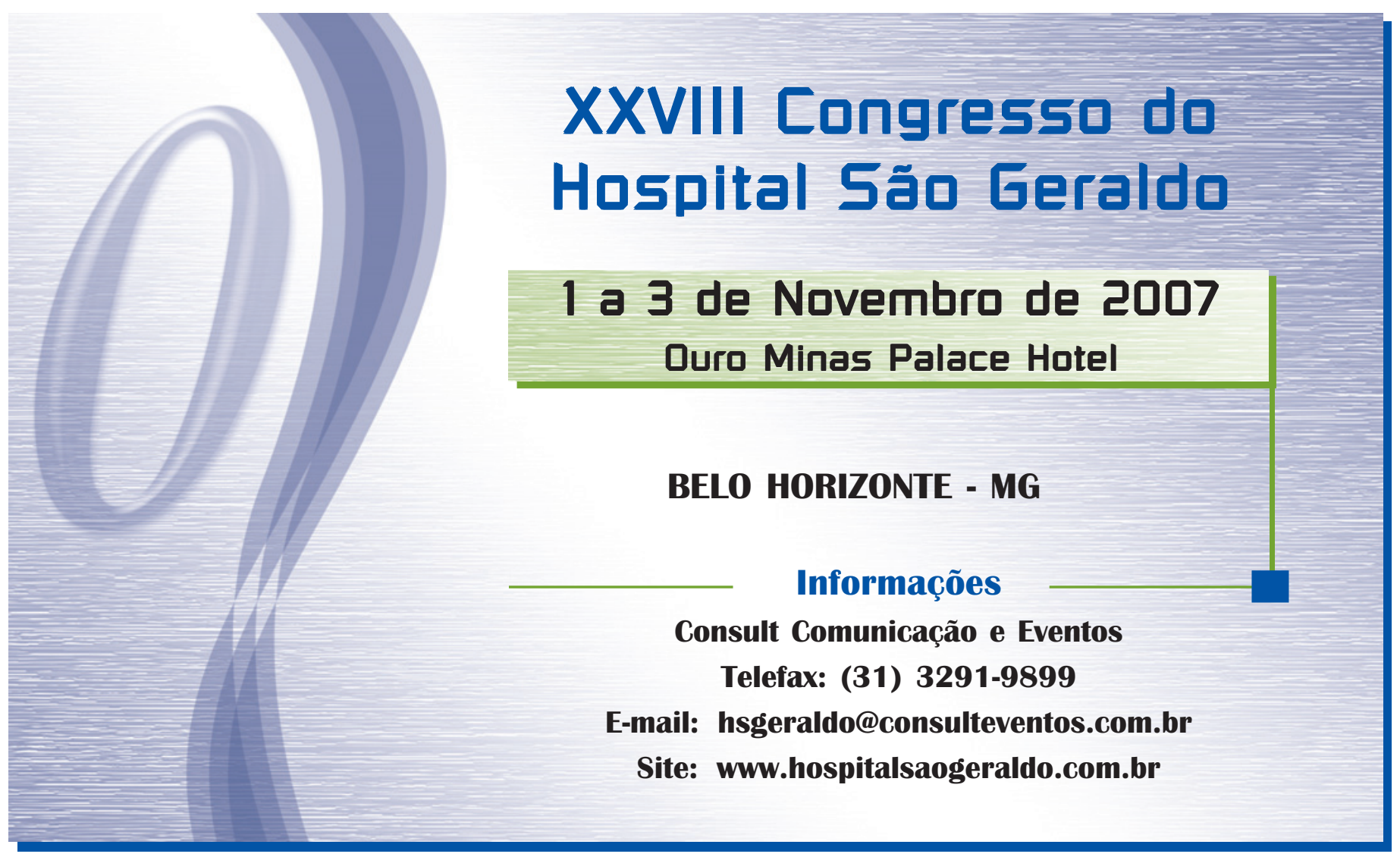

